\title{
Динамика когерентности экситон-поляритонной системы в GaAs-микрорезонаторах при импульсном резонансном фотовозбуждении
}

\author{
(C) В.Д. Кулаковский ${ }^{1,2}$, А.А. Деменев ${ }^{1}$ \\ ${ }^{1}$ Институт фризики твердого тела Российской академии наук, \\ 142432 Черноголовка, Московская обл., Россия \\ ${ }^{2}$ Национальный исследовательский университет „Высшая школа экономики“, \\ 101000 Москва, Россия \\ E-mail: kulakovs@issp.ac.ru
}

Поступила в Редакцию 24 апреля 2019 г.

В окончательной редакции 29 апреля 2019 г.

Принята к публикации 29 апреля 2019 г.

Найдено, что экситон-поляритонные системы в полупроводниковых микрорезонаторах на GaAs, возбуждаемые резонансно когерентными пикосекундными лазерными импульсами, наследуют высокую когерентность лазерного луча и сохраняют ее в течение времени жизни (> 100 пс), при этом время формирования когерентности в системах, возбуждаемых резонансно некогерентными импульсами без возбуждения экситонного резервуара, превышает 200 пс.

Ключевые слова: экситонные поляритоны, бозе-конденсат, пространственная когерентность.

DOI: $10.21883 /$ FTP.2019.10.48287.33

\section{1. Введение}

Квазидвумерные экситонные поляритоны в микрорезонаторах (MP) формируются из экситонов и фотонов в условиях их сильного взаимодействия. Большой интерес к ним обусловлен несколькими причинами: высокой температурой бозе-конденсации вследствие очень малой эффективной массы поляритонов, возможностью контроля величины поляритон-поляритонного взаимодействия и возможностью формирования неравновесных макроскопически когерентных состояний, переходами между которыми можно управлять на масштабах микрометров и пикосекунд [1-5].

Большинство исследований поляритонных конденсатов проводится в условиях межзонного возбуждения, приводящего к образованию плотного экситонного резервуара в квантовых ямах. Поляритоны появляются благодаря рассеянию экситонов на нижнюю поляритонную ветвь. Конденсация поляритонов происходит при плотностях возбуждения выше критической. В недавних исследованиях поляритонных конденсатов было найдено, что в них, как и в классических бозе-эйнштейновских конденсатах, имеют место такие явления, как сверхтекучесть, квантованные вихри [6], тушение зеемановского расщепления [7], эффект Джозефсона [8] и другие. Однако наряду с этим поляритонные конденсаты имеют ряд особенностей, связанных в основном с конечным временем жизни поляритонов и их взаимодействием с экситонным резервуаром, формирующимся при межзонном возбуждении. Так, в исследованиях пространственной когерентности в поляритонных конденсатах при нерезонансном возбуждении было найдено, что она расширяется со скоростью $\sim 10^{8} \mathrm{~cm} / \mathrm{c}$ [9], т. е. на много порядков быстрее, чем в газе ультрахолодных атомов, в то же время длина когерентности не превышает нескольких десятков мкм. Основными причинами нарушения когерентности конденсата, кроме ограниченного времени жизни поляритонов $\tau_{\mathrm{LP}}$, являются потенциальный беспорядок и взаимодействие с экситонным резервуаром $[10,11]$ Поэтому большой интерес представляет исследование „чистой“ поляритонной системы в отсутствие экситонного резервуара.

Возбуждение поляритонной системы без заполнения экситонного резервуара возможно при резонансном возбуждении поляритонов ниже энергии экситона $E_{X}$. При этом возможны два крайних режима: квазистационарное фотовозбуждение импульсами длительностью $t_{p} \gg \tau_{\mathrm{LP}}$ и возбуждение короткими импульсами с $t_{p} \ll \tau_{\mathrm{LP}}$. В первом случае свойства конденсата контролируются возбуждающим светом. При резонансном возбуждении по нормали $(k=0)$ на энергии $\hbar \omega>E_{\mathrm{LP}}$ поляритонная система проявляет мультистабильное поведение [12-15], обусловленное обратной связью в конденсате со спинанизотропным поляритон-поляритонным взаимодействием между амплитудой и эффективной резонансной частотой поля в МР [16,17]. Переключения между стационарными состояниями приводят к таким явлениям, как оптические параметрические осцилляции $[18,19]$, поляритонные солитоны [20-22] спиновые кольца $[13,23]$ и т.д. Во втором случае резонансное возбуждение приводит к образованию поляритонной системы, которая наследует поляризационные и когерентные свойства возбуждающего лазерного луча, а ее дальнейшая динамика определяется временим жизни поляритонов, их межчастичным взаимодействием, а также взаимодействием с фононами и флуктуациями потенциала. 
В этой работе обсуждается динамика когерентных свойств поляритонных систем в GaAs-MP в отсутствие экситонного резервуара, реализуемых при резонансном возбуждении пикосекундными лазерными импульсами ниже энергии экситонного уровня. Рассматриваются случаи возбуждения поляритонной системы на нижнем и верхнем подуровнях нижней поляритонной ветви когерентными гауссовыми импульсами с малой и большой апертурами, а также некогерентными гауссовыми импульсами с большой апертурой.

\section{2. Образцы и экспериментальная методика}

Исследованы 2 структуры МР, выращенные на под-

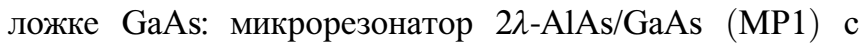
4 наборами из 3 квантовых ям $\operatorname{In}_{0.05} \mathrm{Ga}_{0.95} \mathrm{As}$ толщиной 10 нм и микрорезонатор $\lambda / 2$-AlAs/AlGaAs (MP2) с 4 квантовыми ямами GaAs толщиной 7 нм. Большое количество пар AlAs/GaAs в зеркалах Брэгга обеспечивало добротность $Q>10^{4}$. Расщепление Раби составляло 7.5 мэВ в МР1 и 10.5 мэВ в МР2. В МР2 основное состояние поляритонов было расщеплено на две моды с ортогональными линейными поляризациями $\pi_{x}$ и $\pi_{y}$ из-за анизотропии напряжений в плоскости МР, величина расщепления 80 мкэВ.

Поляритоны возбуждались резонансно с помощью Ті-сапфирового лазера, генерирующего периодические импульсы длительностью $1.5-2.5$ пс с частотой $f=80$ МГц, по нормали к плоскости МР. Генерация поляритонов осуществлялась как в узком диапазоне планарных волновых векторов $\left(|k|<0.2\right.$ мкм $\left.^{-1}\right)$, так и в широком $\left(|k|>0.2\right.$ мкм $\left.^{-1}\right)$. В последнем случае возбуждение осуществлялось сходящимся гауссовым лазерным лучом с большой апертурой $\left(\sim 26^{\circ}\right)$, а большой диаметр возбужденного пятна достигался путем помещения образца на расстоянии $\sim 100$ мкм перед фокусом гауссова пучка. Лазерные импульсы генерировали систему поляритонов выше дна поляритонной ветки $E_{\mathrm{LP}}(k=0)$ на $0.2-0.7 \mathrm{мэВ,} \mathrm{что} \mathrm{на} \mathrm{несколько} \mathrm{мэВ} \mathrm{ниже} \mathrm{энергии}$ экситона $E_{X}$ и достаточно для того, чтобы избежать возбуждения экситонного резервуара.

Образцы находились при гелиевой температуре в оптическом криостате, позволяющем исследовать распределение поляритонов с высоким пространственным $(\sim 1.5$ мкм $)$ и угловым $\left(\sim 1^{\circ}\right)$ разрешениями. Эксперименты проводились при температуре $T=2 \mathrm{~K}$. Сигнал детектировался с помощью стрик-камеры с временны́м разрешением 3 пс.

Пространственная корреляционная функция 1-го порядка $g^{(1)}\left(\mathbf{r}_{1}, \mathbf{r}_{2}, t_{1}-t_{2}=0\right)$ находилась из анализа интерференции излучения поляритонов из разных точек на образце. Эти измерения дают прямую информацию о пространственной когерентности поляритонов, поскольку амплитуда и фаза электрического поля излучения из
МР наследует амплитуду и фазу волновой функции поляритонов. Временна́я эволюция интерферограмм измерялась с использованием интерферометра Майкельсона в режиме, обеспечивающем наложение прямого и зеркального изображений с временны́м разрешением 3 пс.

\section{3. Затухание когерентности экситон-поляритонных систем, возбуждаемых резонансно когерентными пикосекундными импульсами}

\section{1. Поляритонные системы, возбуждаемые по нормали к плоскости микрорезонатора на основном поляритонном подуровне}

Наиболее простую динамику показывают поляритонные системы, возбуждаемые импульсами с $t_{p} \ll \tau_{\mathrm{LP}}$ по нормали к плоскости МР. Нами была исследована динамика такой системы в высокодобротном МР2 с временем жизни поляритонов 19 пс и расщепленным нижним поляритонным уровнем на два подуровня с ортогональной линейной поляризацией. Для возбуждения были использованы когерентные импульсы света с длительно-

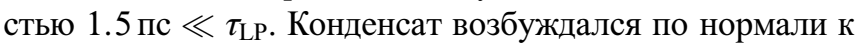
плоскости МР импульсами с $\pi_{y}$-поляризацией, совпадающей с поляризацией нижнего подуровня экситонных поляритонов на энергии $\hbar \omega_{p}=E_{\mathrm{LP}}(k=0)+0.3$ мэВ в пятне диаметром $d=40$ мкм импульсами с энергией 0.12 нДж/импульс при $8 \mathrm{~K}$.

Времяразрешенные поляризационные измерения спектров излучения конденсата показали, что поляритонная система наследует линейную поляризацию возбуждающего импульса. Во время затухания поляритонная система постепенно расширялась из-за отталкивательного межчастичного взаимодействия с сохранением линейной поляризации. Степень деполяризации не превышала $10 \%$ в течение 60 пс.

На рис. 1 показаны временна́я эволюция интерферограмм прямого и зеркального изображений излучения поляритонного конденсата, зарегистрированных с использованием интерферометра Майкельсона из областей с малыми (рис. 1,a) и большими (рис. 1,b) флуктуациями $E_{\mathrm{LP}}$. Из рис. $1, a$ следует, что видность интерференционной картины в конденсате, возбуждаемом в области малых флуктуаций потенциала, почти не изменяется в течение 60 пс, когда интенсивность излучения и, следовательно, плотность конденсата уменьшаются на 2 порядка. Найденные величины корреляционной функции $g^{(1)}(x,-x)$ при $x<D / 2$ из видности интерференционных полос излучения достигают $\sim 0.7$, что близко к измеренному для возбуждаемого лазерного луча $\left(g^{(1)}=0.8\right)$ и к предельному значению величины $g^{(1)}=0.87$ в интерферограммах с периодом осцилляций 7.5 мкм, записанных с пространственным разрешением 2 мкм. 

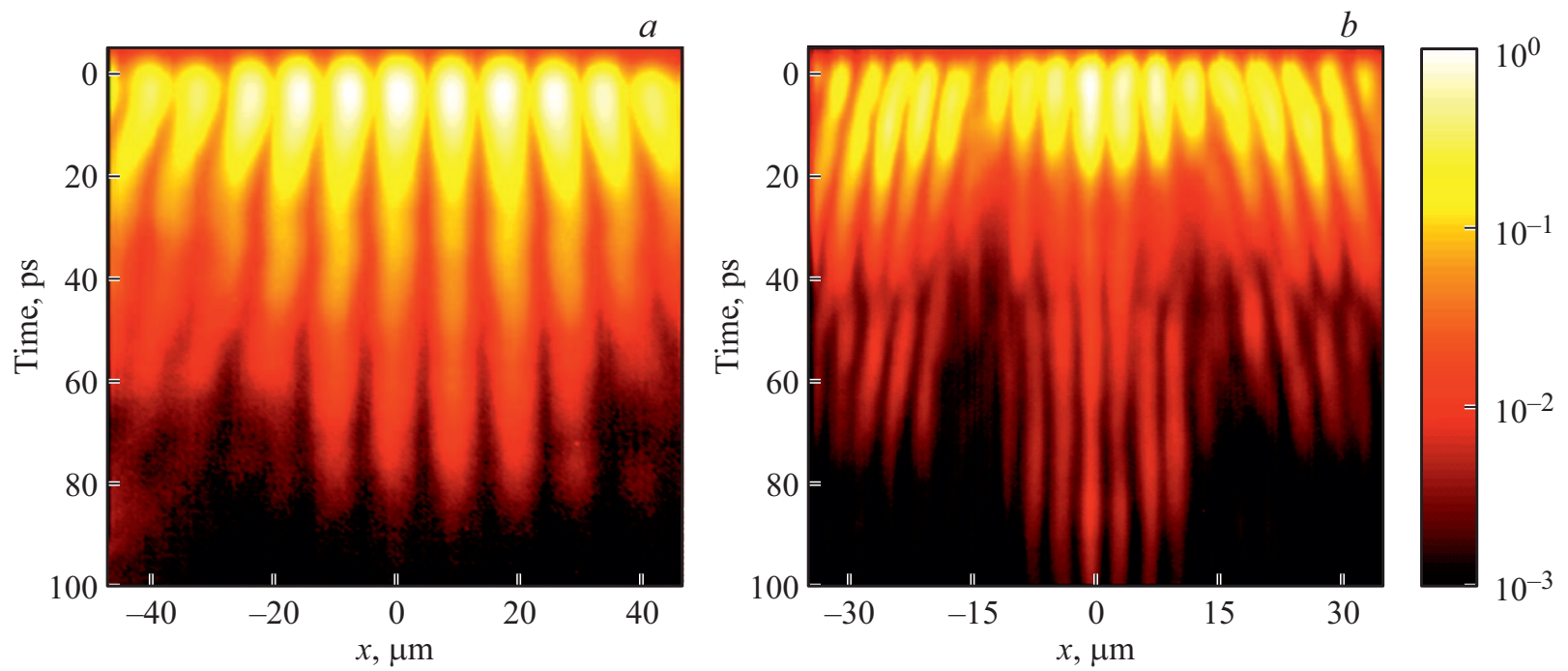

Рис. 1. Временна́я эволюция интерферограмм прямого и зеркального изображений излучения поляритонного конденсата в основном состоянии в MP2 из областей с малыми $(a)$ и большими $(b)$ флуктуациями энергии поляритона, измеренные в области отрицательной расстройки $E_{c}-E_{X}=-6.5$ мэВ и возбуждении на энергии $\hbar \omega_{p}=E_{\mathrm{LP}}(k=0)+0.3$ мэВ импульсами с энергией $P \approx 0.12$ нДж/импульс в пятне диаметром $d=40$ мкм.

Из сравнения интерференционных картин на рис. 1, $a$ и $b$ видно, что в области с большими флуктуациями высокая когерентность также сохраняется в течение $50-60$ пс, даже несмотря на то, что уже при $t \approx 40$ пс происходит разбиение конденсата по локальным минимумам потенциала. Из рис. $1, b$ следует, что когерентность между конденсатами сохраняется в потенцальных ямах на расстоянии $\sim(30-40)$ мкм. Найденная длина когерентности в конденсатах в областях с разными величинами флуктуаций потенциала в несколько раз больше, чем длина когерентности в конденсатах, формирующихся при межзонном возбуждении $[10,11]$. Сохранение когерентности в резонансно возбужденном конденсате поляритонов свидетельствует в пользу выводов работ $[10,11]$ о том, что основной причиной нарушения пространственной когерентности в конденсатах при межзонном возбуждении является его взаимодействие с экситонным резервуаром.

\section{2. Поляритонные системы, возбуждаемые по нормали к плоскости микрорезонатора на верхнем подуровне нижней поляритонной ветви}

Для возбуждения поляритонной системы на верхнем подуровне поляритонной ветви были использованы когерентные лазерные импульсы с линейной поляризацией $\pi_{x}$. На рис. 2 приведены временнб́е зависимости суммарной интенсивности излучения поляритонов, возбужденных импульсами при нормальном падении луча с малой апертурой $\left(<2^{\circ}\right)$ и $\hbar \omega_{p}=E_{\mathrm{LP}}(k=0)+0.7$ мэВ, записанные при энергии накачки $\sim 0.2$ нДж/импульс с разрешением 3 пс, а также зависимости от времени степеней циркулярной $\left(\rho_{c}\right)$, линейных $\left(\rho_{x, y}\right.$ и $\left.\rho_{x \pm y}\right)$ поляризаций излучения и его полной поляризации, $\rho_{\Sigma}=\left(\rho_{x, y}^{2}+\rho_{x \pm y}^{2}+\rho_{c}^{2}\right)^{1 / 2}$.

Из рис. 2 видно, что, как и следовало ожидать, возбуждаемый конденсат наследует поляризацию лазерных импульсов, однако это его поляризационное состояние оказывается неустойчивым: уже при $t \approx 11$ пс $\rho_{x, y}$ уменьшается до нуля. При этом конденсат не переходит в какое-либо определенное стационарное спинорное состояние. Видно, что он переходит в режим джозефсо-

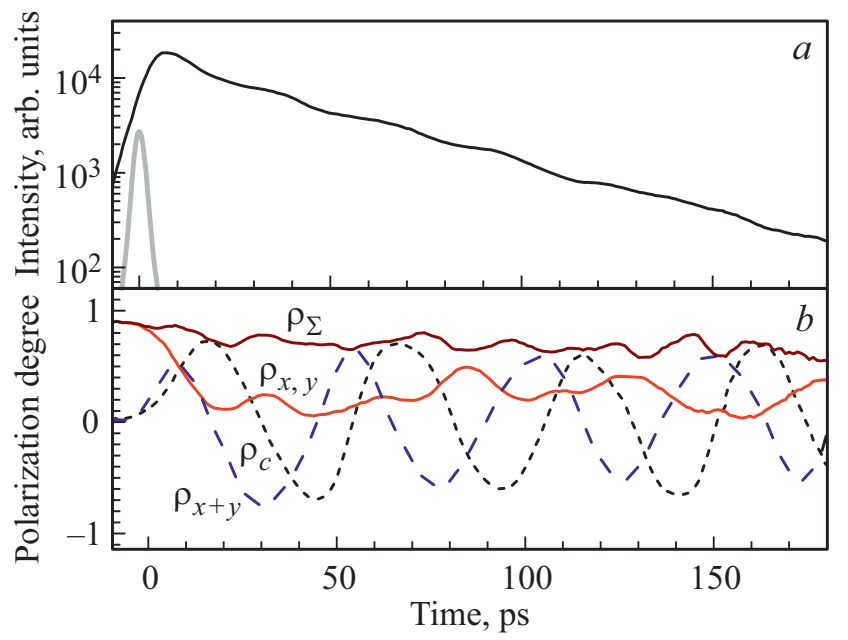

Рис. 2. Временнб́е зависимости суммарной интенсивности излучения поляритонов, записанные с разрешением 3 пс при энергии накачки $\sim 0.2$ нДж/импульс, возбуждении $\pi_{x}$-поляризованными импульсами при нормальном падении луча с малой апертурой $\left(<2^{\circ}\right)$ и $\hbar \omega_{p}=E_{\mathrm{LP}}(k=0)+0.7 \mathrm{мэB}(a)$ и зависимости от времени циркулярной $\left(\rho_{c}\right)$, линейных $\left(\rho_{x, y}\right.$ и $\left.\rho_{x \pm y}\right)$ поляризаций, а также полной поляризации излучения $\left(\rho_{\Sigma}\right)(b)$. 


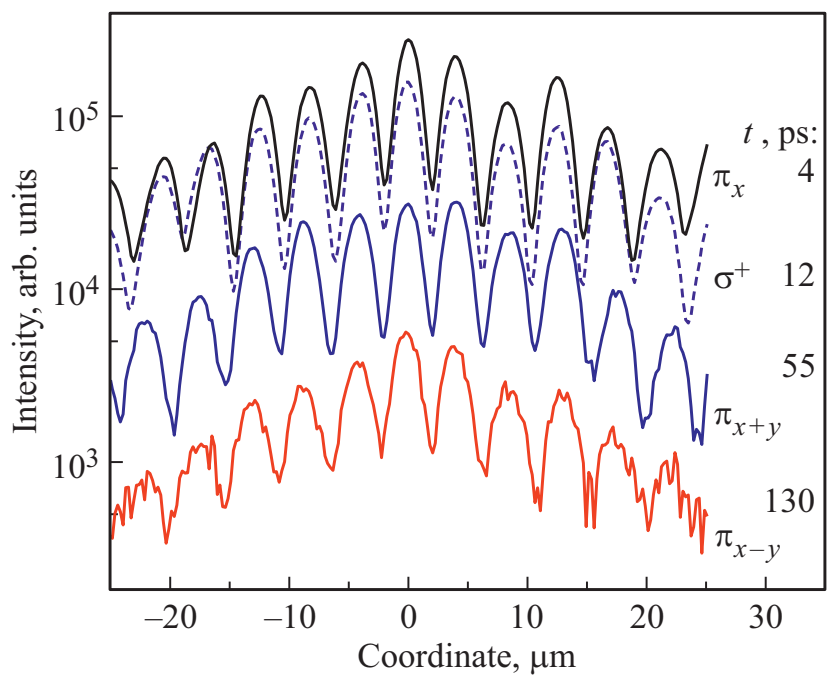

Pис. 3. Эволюция интерферограмм прямого и зеркального изображений излучения поляритонного конденсата на верхнем подуровне в МР2. Поляризации поляритонов и времена задержки указаны.

новских осцилляций с ярко выраженными осцилляциями в двух диагональных линейных $\left(\pi_{x \pm y}\right)$ и в двух круговых $\left(\sigma^{ \pm}\right)$поляризациях. Период осцилляций в обеих поляризациях $T=(47 \pm 1)$ пс соответствуют величине расщепления $\delta_{1}$ уровней $\pi_{x}$ и $\pi_{y} T=h / \delta_{1}=80$ мкэВ в MP2.

Из рис. 2 также следует, что степень полной поляризации конденсата $\rho_{\Sigma}$ изменяется со временем очень слабо. Относительно небольшая потеря полной поляризации конденсата при наличии сильных осцилляций степеней циркулярной и линейных поляризаций свидетельствует о малости некогерентных процессов в конденсате.

Сохранение пространственной когерентности в затухающей спинорной поляритонной системе подтверждается и в измерениях корреляционной функции 1-го порядка излучения конденсата. Интерферограммы прямого и зеркального изображений излучения поляритонного конденсата, записанные в моменты времени, когда конденсат имеет соответственно линейные $\pi_{x}, \pi_{x \pm y}$ и циркулярную $\sigma$ поляризации показаны на рис. 3 .

Из рис. 3 видно, что поляризационная неустойчивость не приводит к нарушению видности интерференционной картины излучения поляритонов и, следовательно, когерентности поляритонного конденсата. Величины $g^{(1)}$ в возбужденном пятне, найденные из интерферограмм, остаются выше 0.5 в течение $\sim 130$ пс, когда плотность поляритонов уменьшается в $\sim 30$ раз, а $\rho_{c}$ и $\rho_{x \pm y}$ имеют 2 периода осцилляций. Таким образом, можно сделать вывод о том, что некогерентное рассеяние поляритонов на фононах и неоднородностях потенциала в поляритонном конденсате, возбуждаемом в $k=0$ на верхнем подуровне поляритонной ветви, так же как и на нижнем, крайне мало.

\section{3. Поляритонные системы, возбуждаемые резонансно когерентным гауссовым пучком в широкой области квазиимпульсов}

Рассмотрим теперь динамику когерентности в поляритонной системе, резонансно возбуждаемой когерентным гауссовым пучком в широкой области $k<1.7 \mathrm{мкм}^{-1}$. Для этой цели был использован сходящийся линейно поляризованный пикосекундный импульс со спектральной шириной $\sim 1.5$ мэВ с большой апертурой. Большой диаметр возбужденного пятна был реализован путем помещения образца на расстоянии 110 мкм перед фокусом. Измерения проводились в MP1 с двукратно вырожденным поляритонным состоянием. Возбуждение осуществлялось на энергии $E_{\mathrm{LP}}(k=0)+0.3$ мэВ широкоапертурным пучком. Поляритоны наследуют планарный квазиимпульс возбуждающего сходящегося луча, их квазиимпульсы направлены к центру, поэтому на первом этапе наблюдается резкое сужение поляритонной системы в координатном пространстве, сопровождающееся торможением поляритонов из-за отталкивательного поляритон-поляритонного взаимодействия и формированием конденсатного состояния на дне поляритонной ветви, а затем его разлетом [24].

Эволюция интерферограмм прямого и зеркального изображений пространственного распределения излучения поляритонов, возбужденных когерентным светом в широком диапазоне энергий, показана на рис. 4. Из рис. 4 следует, что видность интерференционных полос в интерферограммах излучения поляритонов в системе с широким набором квазиимпульсов и энер-

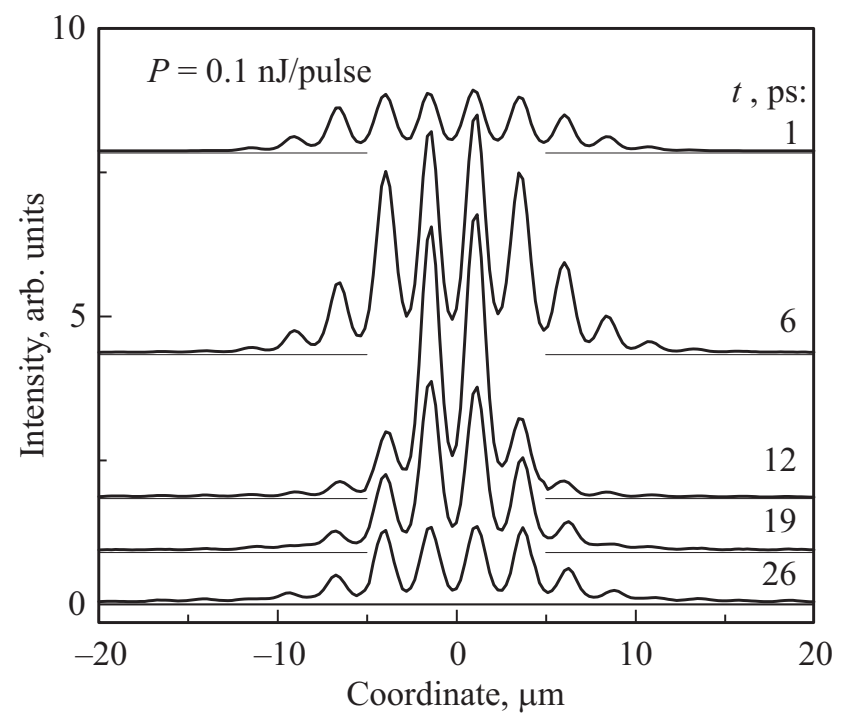

Рис. 4. Временна́я эволюция интерферограмм прямого и зеркального изображений поляритонной системы, возбуждаемой в MP1 широкоапертурным сходящимся когерентным гауссовым пучком по нормали к плоскости МР. МР находится на расстоянии 110 мкм перед фокальной плоскостью. Времена задержки указаны. 
гий поляритонов практически не изменяется как во время динамического сужения поляритонной системы в течение первых $\sim 10$ пс, так и при последующем ее расширении вследствие отталкивательного поляритон-поляритонного взаимодействия.

Таким образом, некогерентное рассеяние поляритонов на фононах в полупроводниковых МР при гелиевых температурах крайне неэффективно даже не только в конденсатном состоянии на дне поляритонной ветви, но и в случае их распределения в широком диапазоне квазиимпульсов. В обоих случаях динамика поляритонной системы практически полностью определяется межчастичным поляритон-поляритонным взаимодействием, которое не нарушает ее когерентность.

\section{4. Поляритонные системы, возбуждаемые некогерентными лазерными импульсами}

Вследствие неэффективности некогерентного поляритон-фононного рассеяния следует ожидать, что установление когерентности в некогерентной поляритонной системе будет происходить очень медленно. Для проверки этого предположения нами было реализовано резонансное возбуждение поляритонной системы с помощью некогерентных импульсов длительностью 2 пс.

Возбуждение осуществлялось в MP1 сходящимся гауссовым лазерным лучом с большой апертурой $\left(\sim 26^{\circ}\right)$ по нормали к плоскости МР. Благодаря большой спектральной полуширине пикосекундных импульсов поляритоны возбуждались в широкой области $k<1.7$ мкм $^{-1}$. Некогерентный свет формировался путем пропускания лазерного луча через матовую кварцевую пластинку. Поляритоны возбуждались при темпе-

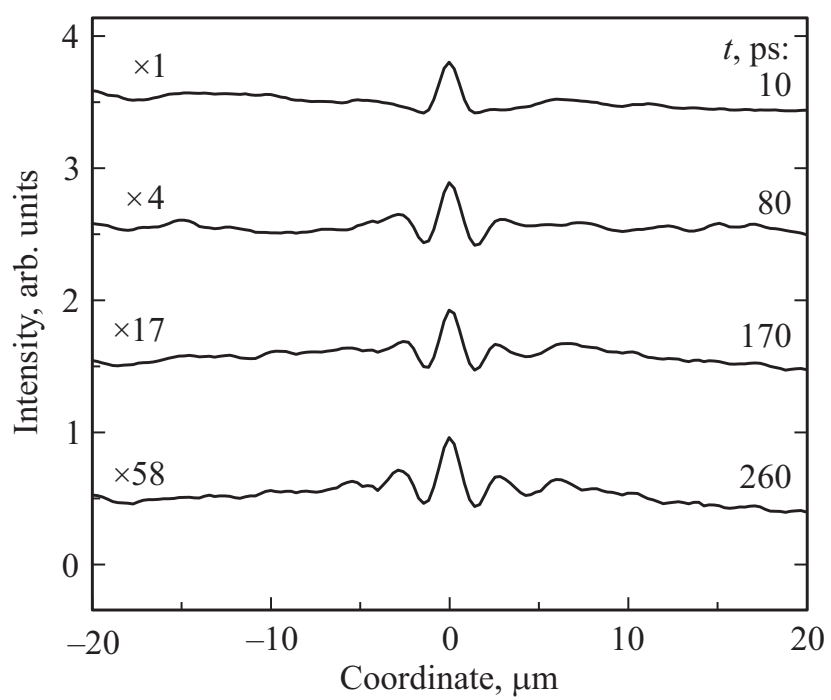

Рис. 5. Временна́я эволюция интерферограмм прямого и зеркального изображений поляритонной системы, возбуждаемой резонансно в MP1 широкоапертурным сходящимся некогерентным гауссовым пучком по нормали к плоскости МР. Времена задержки указаны. ратуре $2 \mathrm{~K}$ в пятне диаметром 80 мкм. Некогерентный свет генерирует поляритоны с широким набором квазиимпульсов во всех точках возбуждаемой области МР, в этом случае не происходит сжатия поляритонной системы. Интенсивность излучения поляритонов затухает со временем их жизни в MP1 $\tau_{\mathrm{LP}} \approx 60$ пс.

Интерферограммы прямого и зеркального изображений излучения затухающей поляритонной системы, возбуждаемой широкоапертурным сходящимся некогерентным гауссовым лучом, приведены на рис. 5 для нескольких времен задержки. Из рисунка видно, что нарастание когерентности в поляритонной системе происходит крайне медленно, интерференционная картина слабо выражена даже на временах 250 пс, величина $g^{(1)}(-x, x)$ при $x=5$ мкм составляет 0.11 и быстро падает с увеличением $x$. Таким образом, в условиях слабого поляритонфононного рассеяния для бозе-эйнштейновской конденсации поляритонов требуются наносекундные времена.

\section{4. Заключение}

Исследована динамика когерентных свойств поляритонных систем в высокодобротных GaAs-MP, реализуемых при резонансном возбуждении пикосекундными лазерными импульсами ниже энергии экситонного уровня без возбуждения экситонного резервуара. Рассмотрены поляритонные системы, возбуждаемые когерентными гауссовыми импульсами с малой и большой апертурами на нижнем и верхнем подуровнях расщепленной нижней поляритонной ветви, а также поляритонные системы, возбуждаемые когерентными и некогерентными гауссовыми импульсами с большой апертурой в MP c вырожденным поляритонным уровнем.

Найдено, что во всех случаях экситон-поляритонные системы наследуют когерентность лазерного луча. Когерентные поляритонные системы сохраняют когерентность в течение времени жизни (> 100 пс), время же формирования когерентности в системах, возбуждаемых резонансно некогерентными импульсами, в отсутствие экситонного резервуара превышает 200 пс.

\section{Благодарности}

Авторы выражают благодарность С.С. Гаврилову, Н.А. Гиппиусу за плодотворные обсуждения и П. Саввидису, С. Хёфлингу за высокодобротные МР-структуры.

\section{Финансирование работы}

Работа выполнена в рамках госзадания Института физики твердого тела РАН с финансовой поддержкой Программы Президиума РАН № 5 „Фотонные технологии в зондировании неоднородных сред и биообъектов“.

\section{Конфликт интересов}

У авторов нет конфликта интересов. 


\section{Список литературы}

[1] J. Kasprzak, J.M. Richard, S. Kundermann, A. Baas, P. Jeambrun, J.M.J. Keeling, F.M. Marchetti, M.H. Szymánska, R. André, J.L. Staehli, V. Savona, P. Littlewood, B. DeveaudPlédran, Le Si Dang. Nature (London, UK), 443, 409 (2006).

[2] R. Balili, V. Hartwell, D. Snoke, L. Pfeiffer, K. West. Science (Washington, DC, US), 316, 1007 (2007).

[3] A.V. Kavokin, J.J. Baumberg, G. Malpuech, F.P. Laussy. Microcavities (Oxford University Press, Oxford, 2007).

[4] D. Sanvitto, V. Timofeev. Exciton Polaritons in Microcavities (Springer, Berlin, 2012).

[5] A. Bramati, M. Modugno. Physics of Quantum Fluids (Springer, Berlin, 2013).

[6] K.G. Lagoudakis, M. Wouters, M. Richard, A. Baas, I. Carusotto, R. André, Le Si Dang, B. Deveaud-Plédran. Nature Phys., 4, 706 (2008).

[7] A.V. Larionov, V.D. Kulakovskii, S. Höfling, C. Schneider, L. Worschech, A. Forchel. Phys. Rev. Lett., 105, 256401 (2010).

[8] K.G. Lagoudakis, B. Pietka, M. Wouters, R. André, B. Deveaud-Plédran. Phys. Rev. Lett., 105, 120403 (2010).

[9] V.V. Belykh, N.N. Sibeldin, V.D. Kulakovskii, M.M. Glazov, M.A. Semina, C. Schneider, S. Höfling, M. Kamp, A. Forchel. Phys. Rev. Lett., 110, 137402 (2013).

[10] Д.А. Мыльников, В.В. Белых, Н.Н. Сибельдин, В.Д. Кулаковский, К. Шнайдер, С. Хефлинг, М. Камп, А. Форхел. Письма ЖЭТФ, 101, 569 (2015).

[11] J. Schmutzler, T. Kazimierczuk, O. Bayraktar, M. Assmann, M. Bayer, S. Brodbeck, M. Kamp, C. Schneider, S. Höfling. Phys. Rev. B, 89, 115119 (2014).

[12] N.A. Gippius, I.A. Shelykh, D.D. Solnyshkov, S.S. Gavrilov, Y. Rubo, A.V. Kavokin, S.G. Tikhodeev, G. Malpuech. Phys. Rev. Lett., 98, 236401 (2007).

[13] I.A. Shelykh, T.C.H. Liew, A.V. Kavokin. Phys. Rev. Lett., 100, 116401 (2008).

[14] T.K. Paraïso, M. Wouters, Y. Leger, F. Morier-Genoud, B. Deveaud-Plédran. Nature Materials, 9, 655 (2010).

[15] S.S. Gavrilov, A.V. Sekretenko, N.A. Gippius, C. Schneider, S. Höfling, M. Kamp, A. Forchel, V.D. Kulakovskii. Phys. Rev. B, 87, 201303(R) (2013).

[16] A.V. Sekretenko, S.S. Gavrilov, V.D. Kulakovskii. Phys. Rev. B, 88, 195302 (2013).

[17] M. Vladimirova, S. Cronenberger, D. Scalbert, K.V. Kavokin, A. Miard, A. Lemaítre, J. Bloch, D. Solnyshkov, G. Malpuech, A.V. Kavokin. Phys. Rev. B, 82, 075301 (2010).

[18] D.N. Krizhanovskii, S.S. Gavrilov, A.P.D. Love, D. Sanvitto, N.A. Gippius, S.G. Tikhodeev, V.D. Kulakovskii, D.M. Whittaker, M.S. Skolnick, J.S. Roberts. Phys. Rev. B, 77, 115336 (2008).

[19] S.S. Gavrilov, A.S. Brichkin, A.A. Demenev, A.A. Dorodnyy, S.I. Novikov, V.D. Kulakovskii, S.G. Tikhodeev, N.A. Gippius. Phys. Rev. B, 85, 075319 (2012).

[20] A.V. Yulin, O.A. Egorov, F. Lederer, D.V. Skryabin. Phys. Rev. A, 78, 061801(R) (2008).

[21] M. Sich, D.N. Krizhanovskii, M.S. Skolnick, A.V. Gorbach, R. Hartley, D.V. Skryabin, E.A. Cerda-Mendez, K. Biermann, R. Hey, P.V. Santos. Nature Photonics, 6, 50 (2012).

[22] G. Slavcheva, A.V. Gorbach, A. Pimenov, A.G. Vladimirova, D.V. Skryabin. Optics Lett., 40, 1787 (2015).
[23] C. Adrados, A. Amo, T.C.H. Liew, R. Hivet, R. Houdre, E. Giacobino, A.V. Kavokin, A. Bramati. Phys. Rev. Lett., 105, 216403 (2010).

[24] В.Д. Кулаковский, С.С. Гаврилов, Н.А. Гиппиус. Письма ЖЭТФ, 106, 605 (2017)

Редактор Л.В. Шаронова

\section{Coherence dynamics of an exciton-polariton fluid in GaAs microcavities under resonant pulsed excitation}

\author{
V.D. Kulakovskii ${ }^{1,2}$, A.A. Demenev ${ }^{1}$ \\ ${ }^{1}$ Institute of Solid State Physics, \\ Russian Academy of Sciences, \\ 142432 Chernogolovka, Moscow Region, Russia \\ ${ }^{2}$ National Research University \\ Higher School of Economics, \\ 101000 Moscow, Russia
}

\begin{abstract}
It was found that exciton-polariton fluids excited resonantly in GaAs semiconductor microcavities by coherent picosecond laser pulses inherit the high coherence of a laser beam and retain it during the lifetime $(>100 \mathrm{ps})$, while the coherence formation time in polariton systems excited resonantly by non-coherent laser pulses without excitation of exciton reservoir exceeds $200 \mathrm{ps}$.
\end{abstract}

